\title{
Applications of Soft Sets in K-Algebras
}

\author{
N. O. Alshehri, ${ }^{1}$ M. Akram, ${ }^{2}$ and R. S. Al-ghamdi ${ }^{1}$ \\ ${ }^{1}$ Department of Mathematics, Faculty of Sciences (Girls), King Abdulaziz University, Jeddah, Saudi Arabia \\ ${ }^{2}$ Punjab University College of Information Technology, University of the Punjab, Old Campus, Lahore 54000, Pakistan
}

Correspondence should be addressed to M. Akram; m.akram@pucit.edu.pk

Received 21 October 2012; Accepted 24 January 2013

Academic Editor: Martine de Cock

Copyright (C) 2013 N. O. Alshehri et al. This is an open access article distributed under the Creative Commons Attribution License, which permits unrestricted use, distribution, and reproduction in any medium, provided the original work is properly cited.

In 1999, Molodtsov introduced the concept of soft set theory as a general mathematical tool for dealing with uncertainty and vagueness. In this paper, we apply the concept of soft sets to $K$-algebras and investigate some properties of Abelian soft $K$-algebras. We also introduce the concept of soft intersection $K$-algebras and investigate some of their properties.

\section{Introduction}

Most of the problems in engineering, medical science, economics, environments, and so forth, have various uncertainties. The problems in system identification involve characteristics which are essentially nonprobabilistic in nature. In response to this situation, Zadeh [1] introduced fuzzy set theory as an alternative to probability theory. Uncertainty is an attribute of information. In order to suggest a more general framework, the approach to uncertainty is outlined by Zadeh [2]. Molodtsov [3] initiated the concept of soft set theory as a new mathematical tool for dealing with uncertainties. In soft set theory, the problem of setting the membership function does not arise, which makes the theory easily applied to many different fields including game theory, operations research, Riemann integration, and Perron integration. At present, work on soft set theory is progressing rapidly. After Molodtsov's work, some operations and application of soft sets were studied by many researchers including Ali et al. [4], Aktaş and Çağman [5], Chen et al. [6], and Maji et al. [7]. Maji et al. [7] gave first practical application of soft sets in decision making problems. To address decision making problems based on fuzzy soft sets, Feng et al. introduced the concept of soft level sets of fuzzy soft sets and initiated an adjustable decision making scheme using fuzzy soft sets [8]. It is interesting to see that soft sets are closely related to many other soft computing models such as rough sets and fuzzy sets. Feng et al. [9] first considered the combination of soft sets, fuzzy sets, and rough sets. Using soft sets as the granulation structures, Feng et al. [10] defined soft approximation spaces, soft rough approximations, and soft rough sets, which are generalizations of Pawlak's rough set model based on soft sets. It has been proven that in some cases Feng's soft rough set model could provide better approximations than classical rough sets. The algebraic structure of soft set theories has been studied increasingly in recent years. Aktaş and Çağman [5] defined the notion of soft groups. Feng et al. [11] initiated the study of soft semirings, and soft rings were defined by Acar et al. [12]. Jun [13] introduced soft $B C K / B C I$-algebras, and Kazancı et al. [14] introduced soft $\mathrm{BCH}$-algebras. Along this direction, we apply soft set theory to $K$-algebras and investigate some of their properties. We introduce the notion of Abelian soft $K$-algebras and investigate some of their properties. We also introduce the concept of soft intersection soft $K$-algebras and investigate some of their properties.

\section{Review of the Literature}

In this section, we recall some basic concepts that are necessary for subsequent discussion of $K$-algebras.

The notion of a $K$-algebra was first introduced by Dar and Akram [15] in 2003 and published in 2005. A $K$-algebra is an algebra built on a group by adjoining an induced binary operation $\odot$ on group which is attached to an abstract $K$-algebra. This system is, in general noncommutative and nonassociative with a right identity $e$, if group is noncommutative. 
Definition 1 (see [15]). Let $(S, \cdot, e)$ be a group in which each nonidentity element is not of order 2 . Then, a $K$-algebra is a structure $\mathscr{K}=(S, \cdot, \odot, e)$ on a group $S$, in which induced binary operation $\odot: S \times S \rightarrow S$ is defined by $\odot(x, y)=$ $x \odot y=x \cdot y^{-1}$ and satisfies the following axioms:

$(K 1)(x \odot y) \odot(x \odot z)=(x \odot((e \odot z) \odot(e \odot y))) \odot x$,

$(K 2) x \odot(x \odot y)=(x \odot(e \odot y)) \odot x$,

$(K 3)(x \odot x)=e$,

$(K 4)(x \odot e)=x$,

$(K 5)(e \odot x)=x^{-1}$

for all $x, y, z \in S$.

Definition 2. A $K$-algebra $\mathscr{K}$ is called abelian if and only if $x \odot(e \odot y)=y \odot(e \odot x)$ for all $x, y \in S$.

If a $K$-algebra $\mathscr{K}$ is abelian, then the axioms $(K 1)$ and (K2) can be written as

$(\overline{K 1})(x \odot y) \odot(x \odot z)=z \odot y$,

$(\overline{K 2}) x \odot(x \odot y)=y$.

In what follows, we denote a $K$-algebra by $\mathscr{K}$ unless otherwise specified.

A nonempty subset $H$ of a $K$-algebra $\mathscr{K}$ is called a subalgebra [15] of the $K$-algebra $\mathscr{K}$ if $a \odot b \in H$ for all $a$, $b \in H$. Note that every subalgebra of a $K$-algebra $\mathscr{K}$ contains the identity $e$ of the group $(G, \cdot, e)$. Naturally, the mapping $f: \mathscr{K}_{1} \rightarrow \mathscr{K}_{2}$ of $K$-algebras is called a homomorphism [16] if $f(x \odot y)=f(x) \odot f(y)$ for all $x, y \in \mathscr{K}_{1}$. We refer the readers to the book [17] and research papers [16-20] for further information regarding $K$-algebras.

Soft Set Theory. In 1999, Molodtsov [3] initiated soft set theory as a new approach for modelling uncertainties. Later on, Maji et al. [21] expanded this theory to fuzzy soft set theory. Based on the idea of parametrization, a soft set gives a series of approximate descriptions of a complicate object from various different aspects. Each approximate description has two parts, namely, predicate and approximate value set. A soft set can be determined by a set-valued mapping assigning to each parameter exactly one crisp subset of the universe. More specifically, we can define the notion of soft set in the following way. Let $U$ be an initial universe and $E$ a set of parameters. Let $P(U)$ denote the power set of $U$, and let $A$ be a nonempty subset of $E$.

Definition 3. A pair $F_{A}=(F, A)$ is called a soft set over $U$, where $A \subseteq E$ and $F: A \rightarrow P(U)$ is a set-valued mapping, called the approximate function of the soft set $F_{A}$. It is easy to represent a soft set $F_{A}$ by a set of ordered pairs as follows:

$$
F_{A}=(F, A)=\{(x, F(x)) \mid x \in A\} .
$$

It is clear to see that a soft set is a parameterized family of subsets of the set $U$. Hereafter unless stated otherwise, we always identify a soft set $F_{A}=(F, A)$ with the corresponding soft set $(F, E)$ with $F(e)=\emptyset$ for all $e \in E \backslash A$. In this fashion, we can implicitly view any soft set as a soft set with the whole parameter set $E$.
Definition 4. Let $F_{A}$ and $G_{B}$ be two soft sets over a common universe $U . F_{A}$ is a said to be a soft subset of $G_{B}$, denoted by $F_{A} \widetilde{\subset} G_{B}$, if $F(x) \subseteq G(x)$ for all $x \in E$.

Definition 5. The restricted intersection of a nonempty family soft sets $\left\{\left(F_{i}\right)_{A_{i}} \mid i \in \Lambda\right\}$ over a common universe $U$ is defined as the soft set $H_{B}=\widetilde{\Pi}_{i \in \Lambda}\left(F_{i}\right)_{A_{i}}$, where $B=\bigcap_{i \in \Lambda} A_{i}$ and $H(x)=\bigcap_{x \in \Lambda} F_{i}(x)$ for all $x \in B$.

Definition 6. The extended intersection of a nonempty family soft sets $\left\{\left(F_{i}\right)_{A_{i}} \mid i \in \Lambda\right\}$ over a common universe $U$ is defined as the soft set $H_{B}=\widetilde{\bigcap}_{i \in \Lambda}\left(F_{i}\right)_{A_{i}}$, where $B=\bigcup_{i \in \Lambda} A_{i}$ and $H(x)=\bigcap_{x \in \Lambda} F_{i}(x), \Lambda(x)=\left\{i \mid i \in A_{i}\right\}$ for all $x \in B$.

Definition 7. The restricted union of a nonempty family soft sets $\left\{\left(F_{i}\right)_{A_{i}} \mid i \in \Lambda\right\}$ over a common universe $U$ is defined as the soft set $H_{B}=\widetilde{\bigcup}_{i \in \Lambda}\left(F_{i}\right)_{A_{i}}$, where $B=\bigcap_{i \in \Lambda} A_{i}$ and $H(x)=$ $\bigcup_{x \in \Lambda} F_{i}(x), \Lambda(x)=\left\{i \mid i \in A_{i}\right\}$ for all $x \in B$.

Definition 8 . The $\wedge$-intersection of a nonempty family soft sets $\left\{\left(F_{i}\right)_{A_{i}} \mid i \in \Lambda\right\}$ over a common universe $U$ is defined as the soft set $H_{B}=\widetilde{\bigwedge}_{i \in \Lambda}\left(F_{i}\right)_{A_{i}}$, where $B=\prod_{i \in \Lambda} A_{i}$ and $H(x)=\bigcap_{x \in \Lambda} F_{i}(x), \Lambda(x)=\left\{i \mid i \in A_{i}\right\}$ for all $x \in B$.

Definition 9. The $\vee$-union of a nonempty family soft sets $\left\{\left(F_{i}\right)_{A_{i}} \mid i \in \Lambda\right\}$ over a common universe $U$ is defined as the soft set $H_{B}=\widetilde{\nabla}_{i \in \Lambda}\left(F_{i}\right)_{A_{i}}$, where $B=\prod_{i \in \Lambda} A_{i}$ and $H(x)=$ $\bigcup_{x \in \Lambda} F_{i}(x), \Lambda(x)=\left\{i \mid i \in A_{i}\right\}$ for all $x \in B$.

Definition 10. The Cartesian product of the nonempty family soft sets $\left\{\left(F_{i}\right)_{A_{i}} \mid i \in \Lambda\right\}$ over a common universe $U$ is defined as the soft set $H_{B}=\widetilde{\prod}_{i \in \Lambda}\left(F_{i}\right)_{A_{i}}$, where $B=\prod_{i \in \Lambda} A_{i}$ and $H(x)=\prod_{x \in \Lambda} F_{i}(x), \Lambda(x)=\left\{i \mid i \in A_{i}\right\}$ for all $x \in B$.

Definition 11. For a soft set $F_{A}$, the set Supp $F_{A}=\{x \in A \mid$ $F(x) \neq \emptyset\}$ is called the support of the soft set $F_{A}$, and the soft set $F_{A}$ is called nonnull if Supp $F_{A} \neq \emptyset$.

We refer the readers to the papers [22-24] for further information regarding the application of soft set theory.

\section{Applications of Soft Sets in K-Algebras}

If $\mathscr{K}$ is a $K$-algebra and $A$ a nonempty set, a set-valued function $F: A \rightarrow \mathscr{P}(\mathscr{K})$ can be defined by $F(x)=\{y \in$ $\mathscr{K} \mid x R y\}, x \in A$, where $R$ is an arbitrary binary relation from $A$ to $\mathscr{K}$; that is, $R$ is a subset of $A \times \mathscr{K}$ unless otherwise specified. The pair $F_{A}$ is then a soft set over $\mathscr{K}$.

Definition 12 (see [19]). Let $F_{A}$ be a soft set over $\mathscr{K}$. Then, $F_{A}$ is called a soft $K$-algebra over $\mathscr{K}$ if $F(x)$ is a $K$-subalgebra of a $K$-algebra $\mathscr{K}$ for all $x \in A$.

Definition 13. Let $F_{A}$ be a nonnull soft set over $\mathscr{K}$. Then, $F_{A}$ is called a soft $K$-algebra over $\mathscr{K}$ if $F(x)$ is a $K$-subalgebra of $\mathscr{K}$ for all $x \in \operatorname{Supp} F_{A}$. 
Example 14. Consider the $K$-algebra $\mathscr{K}=\left(S_{3}, \cdot, \odot, e\right)$ on the symmetric group $S_{3}=\{e, a, b, x, y, z\}$, where $e=(1), a=$ (123), $b=(132), x=(12), y=(13), z=(23)$, and $\odot$ are given by the following Cayley table:

\begin{tabular}{c|cccccc}
$\odot$ & $e$ & $x$ & $y$ & $z$ & $a$ & $b$ \\
\hline$e$ & $e$ & $x$ & $y$ & $z$ & $b$ & $a$ \\
$x$ & $x$ & $e$ & $a$ & $b$ & $z$ & $y$ \\
$y$ & $y$ & $b$ & $e$ & $a$ & $x$ & $z$ \\
$z$ & $z$ & $a$ & $b$ & $e$ & $y$ & $x$ \\
$a$ & $a$ & $z$ & $x$ & $y$ & $e$ & $b$ \\
$b$ & $b$ & $y$ & $z$ & $x$ & $a$ & $e$
\end{tabular}

Let $F_{A}$ be a soft set over $\mathscr{K}$, where $A=\mathscr{K}$ and $F: A \rightarrow P(\mathscr{K})$ is a set-valued function defined by $F(e)=\{e\}, F(a)=F(b)=$ $\{e, a, b\}, F(x)=\{e, x\}, F(y)=\{e, y\}$, and $F(z)=\{e, z\}$ being $K$-subalgebras of $\mathscr{K}$ for all $x \in \operatorname{Supp} F_{A}$. Therefore, $F_{A}$ is a soft $\mathscr{K}$-algebra over $\mathscr{K}$.

Example 15. Consider the $K$-algebra $\mathscr{K}=(G, \cdot, \odot, e)$ on the Dihedral group $G=\{e, a, u, v, b, x, y, z\}$, where $u=a^{2}, v=$ $a^{3}, x=a b, y=a^{2} b, z=a^{3} b$, and $\odot$ are given by the following Cayley table:

\begin{tabular}{c|cccccccc}
$\odot$ & $e$ & $a$ & $u$ & $v$ & $b$ & $x$ & $y$ & $z$ \\
\hline$e$ & $e$ & $v$ & $u$ & $a$ & $b$ & $x$ & $y$ & $z$ \\
$a$ & $a$ & $e$ & $v$ & $u$ & $x$ & $y$ & $z$ & $b$ \\
$u$ & $u$ & $a$ & $e$ & $v$ & $y$ & $z$ & $b$ & $x$ \\
$v$ & $v$ & $u$ & $a$ & $e$ & $z$ & $b$ & $x$ & $y$ \\
$b$ & $b$ & $x$ & $y$ & $z$ & $e$ & $v$ & $u$ & $a$ \\
$x$ & $x$ & $y$ & $z$ & $b$ & $a$ & $e$ & $v$ & $u$ \\
$y$ & $y$ & $z$ & $b$ & $x$ & $u$ & $a$ & $e$ & $v$ \\
$z$ & $z$ & $b$ & $x$ & $y$ & $v$ & $u$ & $a$ & $e$
\end{tabular}

Let $F_{A}$ be a soft set over $\mathscr{K}$, where $A=\mathscr{K}$ and $F: A \rightarrow P(\mathscr{K})$ is set-valued function defined by $F(e)=\{e\}, F(a)=F(v)=$ $\{e, a, u, v\}, F(u)=\{e, u\}, F(x)=\{e, x\}, F(y)=\{e, y\}$, and $F(z)=\{e, z\}$ being $K$-subalgebras of $\mathscr{K}$. Therefore, $F_{A}$ is a soft $K$-algebra over $\mathscr{K}$.

Lemma 16. Let $F_{A}$ be a soft $K$-algebra over $\mathscr{K}$, then

(i) if $x \in F(x) \Rightarrow x^{-1} \in F(x)$ for all $x \in A$,

(ii) if $a \odot b \in F(x) \Rightarrow b \odot a \in F(x)$ for all $a, b \in A$.

Proposition 17. Let $\left\{\left(F_{i}\right)_{A_{i}} \mid i \in \Lambda\right\}$ be a nonempty family of soft $K$-algebras over $\mathscr{K}$. Then, the bi-intersection $\widetilde{\Pi}_{i \in \Lambda}\left(F_{i}\right)_{A_{i}}$ is a soft $K$-algebra over $\mathscr{K}$ if it is nonnull.

Proof. Let $\left\{\left(F_{i}\right)_{A_{i}} \mid i \in \Lambda\right\}$ be a nonempty family of soft $K$ algebras over $\mathscr{K}$. By Definition 5 , we can write $\widetilde{\Pi}_{i \in \Lambda}\left(F_{i}\right)_{A_{i}}=$ $H_{B}$, where $B=\bigcap_{i \in \Lambda} A_{i}$ and $H(x)=\bigcap_{i \in \Lambda} F_{i}(x)$ for all $x \in B$. Let $x \in \operatorname{Supp} H_{B}$. Then, $\bigcap_{i \in \Lambda} F_{i}(x) \neq \emptyset$, and so we have $F_{i}(x) \neq \emptyset$ for all $i \in \Lambda$. Since $\left\{\left(F_{i}\right)_{A_{i}} \mid i \in \Lambda\right\}$ is a nonempty family of soft $K$-algebras over $\mathscr{K}$, it follows that $F_{i}(x)$ is a $K$ subalgebra of $X$ for all $i \in \Lambda$, and its intersection is also a $K$ subalgebra of $\mathscr{K}$; that is, $H(x)=\bigcap_{i \in \Lambda} F_{i}(x)$ is a $K$-subalgebra of $\mathscr{K}$ for all $x \in \operatorname{Supp} H_{B}$. Hence, $H_{B}=\widetilde{\Pi}_{i \in \Lambda}\left(F_{i}\right)_{A_{i}}$ is a soft $K$-algebra over $\mathscr{K}$.
Proposition 18. Let $\left\{\left(F_{i}\right)_{A_{i}} \mid i \in \Lambda\right\}$ be a nonempty family of soft $K$-algebras over $\mathscr{K}$. Then, the extended intersection $\widetilde{\bigcap}_{i \in \Lambda}\left(F_{i}\right)_{A_{i}}$ is a soft $K$-algebra over $\mathscr{K}$.

Proof. Let $\left\{\left(F_{i}\right)_{A_{i}} \mid i \in \Lambda\right\}$ be a nonempty family of soft $K$ algebras over $\mathscr{K}$. By Definition 6 , we can write $\widetilde{\bigcap}_{i \in \Lambda}\left(F_{i}\right)_{A_{i}}=$ $H_{B}$, where $B=\bigcap_{i \in \Lambda} A_{i}$ and $H(x)=\bigcap_{i \in \Lambda} F_{i}(x)$ for all $x \in B$. Let $x \in \operatorname{Supp} H_{B}$. Then, $\bigcap_{i \in \Lambda} F_{i}(x) \neq \emptyset$, and so we have $F_{i}(x) \neq \emptyset$ for all $i \in \Lambda$. Since $\left\{\left(F_{i}\right)_{A_{i}} \mid i \in \Lambda\right\}$ is a nonempty family of soft $K$-algebras over $\mathscr{K}$, it follows that $F_{i}(x)$ is a $K$ subalgebra of $X$ for all $i \in \Lambda$, and its intersection is also a $K$ subalgebra of $\mathscr{K}$; that is, $H(x)=\bigcap_{i \in \Lambda} F_{i}(x)$ is a $K$-subalgebra of $\mathscr{K}$ for all $x \in \operatorname{Supp} H_{B}$. Hence, $H_{B}=\widetilde{\bigcap}_{i \in \Lambda}\left(F_{i}\right)_{A_{i}}$ is a soft $K$-algebra over $\mathscr{K}$.

Proposition 19. Let $\left\{\left(F_{i}\right)_{A_{i}} \mid i \in \Lambda\right\}$ be a nonempty family of soft K-algebras over $\mathscr{K}$. If $F_{i}\left(x_{i}\right) \subseteq F_{j}\left(x_{j}\right)$ or $F_{j}\left(x_{j}\right) \subseteq F_{i}\left(x_{i}\right)$ for all $i, j \in \Lambda, x_{i} \in A_{i}$, then the restricted union $\widetilde{U}\left(F_{i}\right)_{A_{i}}$ is a soft $K$-algebra over $X$.

Proof. Suppose that $\left\{\left(F_{i}\right)_{A_{i}} \mid i \in \Lambda\right\}$ is a nonempty family of soft $K$-algebras over $\mathscr{K}$. By Definition 7 , we can write $\widetilde{\cup}_{i \in \Lambda}\left(F_{i}\right)_{A_{i}}=H_{B}$, where $B=\bigcap_{i \in \Lambda} A_{i}$ and $H(x)=$ $\bigcap_{i \in \Lambda} F_{i}(x)$ for all $x \in B$. Let $x \in \operatorname{Supp} H_{B}$. Since Supp $H_{B}=\bigcup_{i \in \Lambda} \operatorname{Supp}\left(F_{i}\right)_{A_{i}} \neq \emptyset, F_{i_{0}}(x) \neq \emptyset$ for some $i_{0} \in \Lambda$. By assumption, $\bigcup_{i \in \Lambda} F_{i}(x)$ is a $K$-subalgebra of $\mathscr{K}$ for all $x \in \operatorname{Supp} H_{B}$. Hence, restricted union $\widetilde{\bigcup}_{i \in \Lambda}\left(F_{i}\right)_{A_{i}}$ is a soft $K$ algebra over $\mathscr{K}$.

Proposition 20. Let $\left\{\left(F_{i}\right)_{A_{i}} \mid i \in \Lambda\right\}$ be a nonempty family of soft $K$-algebras over $\mathscr{K}$. Then, the $\wedge$-intersection $\widetilde{\bigwedge}_{i \in \Lambda}\left(F_{i}\right)_{A_{i}}$ is a soft $K$-algebra over $\mathscr{K}$ if it is nonnull.

Proof. Let $\left\{\left(F_{i}\right)_{A_{i}} \mid i \in \Lambda\right\}$ be a nonempty family of soft $K$ algebras over $\mathscr{K}$. By Definition 11, we can write $\widetilde{\bigwedge}_{i \in \Lambda}\left(F_{i}\right)_{A_{i}}=$ $H_{B}$, where $B=\prod_{i \in \Lambda} A_{i}$ and $H(x)=\bigcap_{i \in \Lambda} F_{i}(x)$ for all $x=$ $\left(x_{i}\right)_{i \in \Lambda} \in B$. Suppose that the soft set $H_{B}$ is nonempty. If $x=$ $\left(x_{i}\right)_{i \in \Lambda} \in \operatorname{Supp} H_{B}, H(x)=\bigcap_{i \in \Lambda} F_{i}(x) \neq \emptyset$. Since $\left\{\left(F_{i}\right)_{A_{i}} \mid i \in\right.$ $\Lambda\}$ is a nonempty family of soft $K$-algebras over $\mathscr{K}$, nonempty set $F_{i}(x)$ is a $K$-subalgebra of $\mathscr{K}$ for all $i \in \Lambda$. It follows that $H(x)=\bigcap_{i \in \Lambda} F_{i}(x)$ is a $K$-subalgebra of $\mathscr{K}$ for all $x=\left(x_{i}\right)_{i \in \Lambda} \in$ Supp $H_{B}$. Hence $\wedge$-intersection $\widetilde{\bigwedge}_{i \in \Lambda}\left(F_{i}\right)_{A_{i}}$ is a soft $K$-algebra over $\mathscr{K}$.

Proposition 21. Let $\left\{\left(F_{i}\right)_{A_{i}} \mid i \in \Lambda\right\}$ be a nonempty family of soft K-algebras over $\mathscr{K}$. If $F_{i}\left(x_{i}\right) \subseteq F_{j}\left(x_{j}\right)$ or $F_{j}\left(x_{j}\right) \subseteq F_{i}\left(x_{i}\right)$ for all $i, j \in \Lambda, x_{i} \in A_{i}$, then the $\vee$-union $\widetilde{\nabla}\left(F_{i}\right)_{A_{i}}$ is a soft $K$-algebra over $\mathscr{K}$.

Proof. Assume that $\left\{\left(F_{i}\right)_{A_{i}} \mid i \in \Lambda\right\}$ is a nonempty family of soft $K$-algebras over $\mathscr{K}$. By Definition 9 , we can write $\widetilde{\nabla}_{i \in \Lambda}\left(F_{i}\right)_{A_{i}}=H_{B}$, where $B=\prod_{i \in \Lambda} A_{i}$ and $H(x)=\bigcup_{i \in \Lambda} F_{i}(x)$ for all $x=\left(x_{i}\right)_{i \in \Lambda} \in B$. Let $x=\left(x_{i}\right)_{i \in \Lambda} \in \operatorname{Supp} H_{B}$. Then, $H(x)=\bigcup_{i \in \Lambda} F_{i}(x) \neq \emptyset$, so we have $F_{i_{0}}\left(x_{i_{0}}\right) \neq \emptyset$ for some $i_{i_{0}} \in$ $\Lambda$. By assumption, $\bigcup_{i \in \Lambda} F_{i}(x)$ is a $K$-subalgebra of $\mathscr{K}$ for all $x=\left(x_{i}\right)_{i \in \Lambda} \in \operatorname{Supp} H_{B}$. Hence, $\vee$-union $\widetilde{\nabla}\left(F_{i}\right)_{A_{i}}$ is a soft $K$ algebra over $\mathscr{K}$. 
Proposition 22. Let $\left\{\left(F_{i}\right)_{A_{i}} \mid i \in \Lambda\right\}$ be a nonempty family of soft $K$-algebras over $\mathscr{K}$. Then, the cartesian product $\widetilde{\prod}_{i \in \Lambda}\left(F_{i}\right)_{A_{i}}$ is a soft $K$-algebra over $\prod_{i \in \Lambda} \mathscr{K}_{i}$.

Proof. Let $\left\{\left(F_{i}\right)_{A_{i}} \mid i \in \Lambda\right\}$ be a nonempty family of soft $K$ algebras over $\mathscr{K}$. By Definition 10 , we can write $\widetilde{\prod}_{i \in \Lambda}\left(F_{i}\right)_{A_{i}}=$ $H_{B}$, where $B=\prod_{i \in \Lambda} A_{i}$ and $H(x)=\prod_{i \in \Lambda} F_{i}(x)$ for all $x=\left(x_{i}\right)_{i \in \Lambda} \in B$. Suppose that the soft set $H_{B}$ is nonnull. If $x=\left(x_{i}\right)_{i \in \Lambda} \in \operatorname{Supp} H_{B}, H(x)=\prod_{i \in \Lambda} F_{i}(x) \neq \emptyset$. Since $\left\{\left(F_{i}\right)_{A_{i}} \mid i \in \Lambda\right\}$ is a nonempty family of soft $K$-algebras over $\mathscr{K}$, the nonempty set $F_{i}(x)$ is a $K$-subalgebra of $\mathscr{K}$ for all $i \in \Lambda$. It follows that $H(x)=\prod_{i \in \Lambda} F_{i}(x)$ is a $K$-subalgebra of $\mathscr{K}$ for all $x=\left(x_{i}\right)_{i \in \wedge} \in \operatorname{Supp} H_{B}$. Hence, $\wedge$-intersection $\widetilde{\prod}_{i \in \Lambda}\left(F_{i}\right)_{A_{i}}$ is a soft $K$-algebra over $\prod_{i \in \Lambda} \mathscr{K}_{i}$.

Definition 23. Let $F_{A}$ be a soft $K$-algebra over $\mathscr{K}$.

(i) $F_{A}$ is called the trivial soft $K$-algebra over $\mathscr{K}$ if $F(x)=$ $\{e\}$ for all $x \in A$.

(ii) $F_{A}$ is called the whole soft $K$-algebra over $\mathscr{K}$ if $F(x)=$ $\mathscr{K}$ for all $x \in A$.

Definition 24. Let $F_{A}$ be a soft set over a $K$-algebra $\mathscr{K}$. Then, the inverse of $F_{A}$ is denoted by $F_{A}^{-1}$ and is defined as follows $F_{A}^{-1}=\left\{(F(a))^{-1}: a \in A\right\}$, where $(F(a))^{-1}$ is called the inverse of $F(a)$ and is defined as $(F(a))^{-1}=\left\{x^{-1}: x \in F(a)\right\}$

Theorem 25. Let $F_{A}$ and $G_{B}$ be any two soft sets over $\mathscr{K}$. Then $\left(F_{A} \widehat{\circ} G_{B}\right)^{-1}=G_{B}^{-1} \widehat{\circ} F_{A}^{-1}$.

Theorem 26. If $F_{A}$ is a soft $K$-algebra over $\mathscr{K}$, then $F_{A}^{-1}=F_{A}$.

The converse of above theorem is not true in general, and it can be seen in the following example.

Example 27. Consider the $K$-algebra $K=(G, \cdot, \odot, e)$ on the dihedral group $G=\{e, a, u, v, b, x, y, z\}$ which is given in Example 15. Let $F_{A}$ be a soft set over $\mathscr{K}$, where $A=\mathscr{K}$ and $F: A \rightarrow P(\mathscr{K})$ is a set-valued function defined by $(F(e))^{-1}=$ $\{e\},(F(a))^{-1}=\{e, a, u\},(F(u))^{-1}=\{e, u\},(F(v))^{-1}=\{e, v, u\}$, $(F(b))^{-1}=\{e, b),(F(x))^{-1}=\{e, x\},(F(y))^{-1}=\{e, y\}$, and $(F(z))^{-1}=\{e, z\}$. Therefore, we find that $F(\alpha)=(F(\alpha))^{-1}$ for all $\alpha \in A$. Hence $F_{A}^{-1}=F_{A}$, but $F_{A}$ is not soft $K$-algebra over $\mathscr{K}$ because $F(a)=\{e, a, u\}$ is not a $K$-subalgebra of $K$.

Definition 28. A soft $K$-algebra $F_{A}$ over $\mathscr{K}$ is said to be Abelian soft $K$-algebra over $\mathscr{K}$ if each $F(\alpha)$ is an Abelian $K$ subalgebra of $\mathscr{K}$ for all $\alpha \in A$.

Example 29. Let $F_{A}$ be a soft $K$-algebra over $\mathscr{K}$, which is given in Example 14. Then, it is easy to verify that each $F(x)$ is an Abelian $K$-subalgebra of $K$ for all $x \in A$. Hence, $F_{A}$ is an Abelian soft $K$-algebra over $K$.

Definition 30. Let $F_{A}$ be a soft $K$-algebra over $\mathscr{K}$ and $H_{B}$ a soft $K$-subalgebra of $F_{A}$. Then, we say that $H_{B}$ is an Abelian soft $K$-subalgebra of $F_{A}$ if $H(x)$ is an Abelian $K$-subalgebra of $F(x)$ for all $x \in B$.
Example 31. Let $F_{A}$ be a soft $K$-algebra over $\mathscr{K}$ which is given in Example 14, and let $H_{B}$ be a soft set over $\mathscr{K}$, where $B=$ $A_{3}$ and $H: B \rightarrow P(K)$ is the set-valued function defined by $H(e)=\{e\}, H(a)=\{e, a, b\}$, and $H(b)=\{e, a, b\}$ being Abelian $K$-subalgebras of $F(e), F(a)$, and $F(b)$, respectively. Hence, $H_{B}$ is an Abelian soft $K$-subalgebra of $F_{A}$.

Theorem 32. Let $F_{A}$ be an Abelian soft $K$-algebra over $\mathscr{K}$ and $G_{B}$ be a soft $K$-algebras over $\mathscr{K}$. Then their restricted intersection $F_{A} \widetilde{\sqcap} G_{B}$ is an Abelian soft $K$-algebra over $\mathscr{K}$ for all $c \in A \cap B$.

Definition 33. Let $\mathscr{K}_{1}, \mathscr{K}_{2}$ be two $K$-algebras and $\varphi: \mathscr{K}_{1} \rightarrow$ $\mathscr{K}_{2}$ a mapping of $K$-algebras. If $F_{A}$ and $G_{B}$ are soft sets over $\mathscr{K}_{1}$ and $\mathscr{K}_{2}$, respectively, then $\varphi\left(F_{A}\right)$ is a soft set over $\mathscr{K}_{2}$ where $\varphi(F): E \rightarrow P\left(\mathscr{K}_{2}\right)$ is defined by $\varphi(F)(x)=\varphi(F(x))$ for all $x \in E$ and $\varphi^{-1}\left(G_{B}\right)$ is a soft set over $\mathscr{K}_{1}$ where $\varphi^{-1}(G)$ : $E \rightarrow P\left(\mathscr{K}_{1}\right)$ is defined by $\varphi^{-1}(G)(y)=\varphi^{-1}(G(y))$ for all $y \in E$.

Definition 34. Let $F_{A}$ and $H_{B}$ be two soft sets over $K$-algebras $\mathscr{K}_{1}$ and $\mathscr{K}_{2}$, respectively, and let $\varphi: \mathscr{K}_{1} \rightarrow \mathscr{K}_{2}$ and $\phi:$ $A \rightarrow B$ be two functions. Then, we say that $(\varphi, \phi)$ is a soft homomorphism, if the following conditions are satisfied:

(i) $\varphi$ is a homomorphism from $\mathscr{K}_{1}$ onto $\mathscr{K}_{2}$;

(ii) $\phi$ is mapping from $A$ onto $B$;

(iii) $\varphi(F(x))=H(\phi(x))$.

In this definition, if $\varphi$ is an isomorphism $\mathscr{K}_{1}$ to $\mathscr{K}_{2}$ and $\phi$ is a one-to-one mapping from $A$ on to $B$, then we say that $(\varphi, \phi)$ is a soft isomorphism and that $F_{A}$ is soft isomorphic to $H_{B}$. Notation, $F_{A} \simeq H_{B}$.

Example 35. Let $S=\{e, a, b, c\}$ be a Klein four-group. Consider a $K$-algebra on $G$ with the following Cayley table:

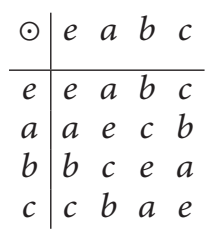

This is an improper $K$-algebra on Klein four-group since it is an elementary Abelian 2-group, that is, $x \odot y=x \cdot y^{-1}=$ $x \cdot y$. Let $F_{A}$ be a soft set over $\mathscr{K}$, where $A=\mathscr{K}$ and $F$ : $A \rightarrow P(\mathscr{K})$; the set-valued function defined by $F(x)=\{r \in$ $\left.\mathscr{K}: x R r \Leftrightarrow x \odot r \in A_{x}\right\}$ where $A_{x}=\left\{e, x, x^{-1}\right\}$. Then, $F_{A}$ is a soft $\mathscr{K}$-algebra over $\mathscr{K}$. Let $(G, A)$ be a soft set over $\mathscr{K}$, where $A=\mathscr{K}$ and $G: A \rightarrow P(\mathscr{K})$ is the set-valued function defined by $G(x)=\left\{r \in \mathscr{K}: x R r \Leftrightarrow x^{n}=r, n \in \mathbb{N}\right\}$. Then $G_{A}$ is a soft $\mathscr{K}$-algebra over $\mathscr{K}$. Let $\varphi: \mathscr{K} \rightarrow \mathscr{K}$ be the mapping defined by $\varphi(x)=x$. It is clear that $\varphi$ is a $\mathscr{K}$-homomorphism. Consider the mapping $\phi: A \rightarrow A$ given by $\phi(x)=x^{3}$. Then, one can easily verify that $\varphi(F(x))=G(\phi(x))$ for all $x \in A$. Hence, $(\varphi, \phi)$ is a soft homomorphism from $\mathscr{K}$ to $\mathscr{K}$. 
Example 36. Consider the $K$-algebra $K=\left(S_{3}, \cdot, \odot, e\right)$ which is given in Example 14. Let $F_{A}$ be a soft set over $\mathscr{K}$, where $A=A_{3} \subset K$ and $F: A \rightarrow P(\mathscr{K})$ is the set-valued function defined by $F(x)=\left\{r \in \mathscr{K}: x R r \Leftrightarrow x \odot r \in A_{x}\right\}$, where $A_{x}=\left\{e, x, x^{-1}\right\}$. Then, $F_{A}$ is a soft $K$-algebra over $\mathscr{K}$. Let $G_{A}$ be a soft set over $\mathscr{K}$, where $A=A_{3}$ and $G: A \rightarrow P(\mathscr{K})$ is the set-valued function defined by $G(x)=\left\{r \in \mathscr{K}: x R r \Leftrightarrow x^{n}=\right.$ $r, n \in \mathbb{N}\}$. Then, $G_{A}$ is a soft $K$-algebra over $\mathscr{K}$. Let $f: \rightarrow \mathscr{K}$ be the mapping defined by $\varphi(x)=x^{4}$. It is clear that $\varphi$ is a $K$ homomorphism. Consider the mapping $\phi: A \rightarrow A$ given by $\phi(x)=x$. Then, one can easily verify that $\varphi(F(x))=G(\phi(x))$ for all $x \in A$. Hence, $(\varphi, \phi)$ is a soft homomorphism from $\mathscr{K}$ to $\mathscr{K}$.

We state the following propositions without their proofs.

Proposition 37. Let $f: \mathscr{K}_{1} \rightarrow \mathscr{K}_{2}$ be an onto homomorphism of $K$-algebras and $F_{A}, G_{B}$ two soft $K$-algebras over $\mathscr{K}_{1}$ and $\mathscr{K}_{2}$, respectively.

(i) The soft function $\left(\varphi, I_{A}\right)$ from $F_{A}$ to $(H, A)$ is a soft homomorphism from $\mathscr{K}_{1}$ to $\mathscr{K}_{2}$, where $I_{A}: A \rightarrow A$ is the identity mapping and the set-valued function $H: A \rightarrow P\left(\mathscr{K}_{2}\right)$ is defined by $H(x)=\varphi(F(x))$ for all $x \in A$.

(ii) If $\varphi \quad: \mathscr{K}_{1} \rightarrow \mathscr{K}_{2}$ is an isomorphism, then the soft function $\left(f^{-1}, I_{B}\right)$ from $G_{B}$ to $S_{B}$ is a soft homomorphism from $\mathscr{K}_{2}$ to $\mathscr{K}_{1}$, where $I_{B}: B \rightarrow B$ is the identity mapping and the set-valued function $S: B \rightarrow P\left(\mathscr{K}_{1}\right)$ is defined by $S(x)=\varphi^{-1}(G(x))$ for all $x \in B$.

Proposition 38. Let $\mathscr{K}_{1}, \mathscr{K}_{2}$, and $\mathscr{K}_{3}$ be $K$-algebras and $F_{A}, G_{B}$ and $H_{C}$ soft $K$-algebras over $\mathscr{K}_{1}, \mathscr{K}_{2}$, and $\mathscr{K}_{3}$, respectively. Let the soft function $(\varphi, \phi)$ from $F_{A}$ to $G_{B}$ be a soft homomorphism from $\mathscr{K}_{1}$ to $\mathscr{K}_{2}$, and the soft function $\left(f^{\prime}, g^{\prime}\right)$ from $G_{B}$ to $H_{C}$ a soft homomorphism from $\mathscr{K}_{2}$ to $\mathscr{K}_{3}$. Then, the soft function $\left(\varphi^{\prime} \circ \varphi, \phi^{\prime} \circ \phi\right)$ from $F_{A}$ to $H_{C}$ is a soft homomorphism from $\mathscr{K}_{1}$ to $\mathscr{K}_{3}$.

Theorem 39. Let $\mathscr{K}_{1}$ and $\mathscr{K}_{2}$ be $K$-algebras and $F_{A}, G_{B}$ soft sets over $\mathscr{K}_{1}$ and $\mathscr{K}_{2}$, respectively. If $F_{A}$ is a soft $K$-algebra over $\mathscr{K}_{1}$ and $F_{A} \simeq G_{B}$, then $G_{B}$ is a soft $K$-algebra over $\mathscr{K}_{2}$.

Definition 40. Let $F_{A}$ and $H_{B}$ be two soft $K$-algebras over $\mathscr{K}_{1}$ and $\mathscr{K}_{2}$, respectively. Then the Cartesian product of soft $K$ algebras $F_{A}$ and $H_{B}$ is denoted by $F_{A} \widetilde{\times} H_{B}=(U, A \times B)$ and $U$ is defined as $U(a, b)=F(a) \times H(b)$ for all $(a, b) \in A \times B$.

Theorem 41. Let $F_{A}$ and $H_{B}$ be two soft $K$-algebras over $\mathscr{K}_{1}$ and $\mathscr{K}_{2}$, respectively. Then, (1) the Cartesian product $F_{A} \widetilde{\times} H_{B}$ is a soft $K$-algebra over $\mathscr{K}_{1} \times \mathscr{K}_{2}$, and (2) $F_{A} \widetilde{\times} H_{B}$ is soft isomorphic to $H_{B} \widetilde{\times} F_{A}$.

Proof. We will prove second part. We show that $(\varphi, \phi)$ : $F_{A} \tilde{\times} H_{B} \rightarrow H_{B} \widetilde{\times} F_{A}$ is a soft isomorphism, that is, $(\varphi, \phi)$ : $(U, A \times B) \rightarrow(W, B \times A)$ is a soft isomorphism where $W(b, a)$ is defined as $W(b, a)=H(b) \times F(a)$. We prove the following three conditions:

(i) we show that $\varphi: \mathscr{K}_{1} \times \mathscr{K}_{2} \rightarrow \mathscr{K}_{2} \times \mathscr{K}_{1}$ is an isomorphism. Let $\varphi$ be a function defined by $\varphi(r, s)=$ $(s, r)$. Then obviously $\varphi$ is an isomorphism.

(ii) We now show that $\phi: A \times B \rightarrow B \times A$ is a bijective mapping. The mapping $\phi$ is defined by $\phi(a, b)=(b, a)$ then obviously $\phi$ is a bijective mapping.

(iii) Consider

$$
\begin{aligned}
\varphi(U(a, b)) & =\varphi(F(a) \times H(b)) \\
& =\varphi(\{(r, s): r \in F(a), s \in H(b)\}) \\
& =\{(s, r): s \in H(b), r \in F(a)\} \\
& =H(b) \times F(a) \\
& =W(b, a)=W(g(a, b))
\end{aligned}
$$

for all $(a, b) \in A \times B$. This implies that $(\varphi, \phi): F_{A} \tilde{\times} H_{B} \rightarrow$ $H_{B} \widetilde{\times} F_{A}$ is a soft isomorphism. Hence, $F_{A} \widetilde{\times} H_{B} \simeq H_{B} \widetilde{\times} F_{A}$.

We now introduce the concept of soft intersection soft $K$ algebras and investigate some of their properties

Definition 42 . Let $\mathscr{K}=E$ be a $K$-algebra and let $A$ be a subset of $\mathscr{K}$. Let $F_{A}$ be a soft set over $U$. Then, $F_{A}$ is called a soft intersection $K$-subalgebra over $U$ if it satisfies the following condition:

$$
F(x \odot y) \supseteq F(x) \cap F(y)
$$

for all $x, y \in A$.

Example 43. Assume that $U=\mathbb{Z}$ is the universal set. Let $A=$ $G=\left\{e, a, a^{2}\right\}$ be the cyclic group of order 3 . Then, $(G, \cdot, \odot, e)$ is a $K$-algebra $\mathscr{K}$, and $\odot$ is given by the following Cayley table:

\begin{tabular}{c|ccc}
$\odot$ & $e$ & $a$ & $a^{2}$ \\
\hline$e$ & $e$ & $a^{2}$ & $a$ \\
$a$ & $a$ & $e$ & $a^{2}$ \\
$a^{2}$ & $a^{2}$ & $a$ & $e$
\end{tabular}

Let $F_{A}$ be a soft set over $U$. Then, $F(e)=\mathbb{Z}$ and $F(a)=F\left(a^{2}\right)=$ $\{-2,-1,0,1,2\}$. It is easy to see that $F_{A}$ is a soft intersection $K$ subalgebra over $U$.

From now on, we will always assume that $\mathscr{K}=E$ unless otherwise specified.

Proposition 44. Let $\mathscr{K}$ be a K-algebra and let $A$ and $B$ be $K$-subalgebras of $\mathscr{K}$. If $F_{A}$ and $G_{B}$ are soft intersection $K$-subalgebras over $U$. Then, $F_{A} \tilde{\wedge} G_{B}$ is a soft intersection $K$-subalgebra over $U$, where $F_{A} \widetilde{\wedge} G_{B}$ is defined by $F_{A} \tilde{\wedge} G_{B}(x, y)=F(x) \cap G(y)$ for all $(x, y) \in A \times B$. 
Proof. Let $\left(x_{1}, y_{1}\right),\left(x_{2}, y_{2}\right) \in A \times B$. Then,

$$
\begin{aligned}
& \left(F_{A} \tilde{\wedge} G_{B}\right)\left(\left(x_{1}, y_{1}\right) \odot\left(x_{2}, y_{2}\right)\right) \\
& \quad=\left(F_{A} \tilde{\wedge} G_{B}\right)\left(\left(x_{1} \odot x_{2}, y_{1} \odot y_{2}\right)\right) \\
& \quad=F\left(x_{1} \odot x_{2}\right) \cap G\left(y_{1} \odot y_{2}\right) \\
& \quad \supseteq\left(F\left(x_{1}\right) \cap F\left(x_{2}\right)\right) \cap\left(G\left(y_{1}\right) \cap G\left(y_{2}\right)\right) \\
& \quad=\left(F\left(x_{1}\right) \cap G\left(y_{1}\right)\right) \cap\left(F\left(x_{2}\right) \cap G\left(y_{2}\right)\right) \\
& \quad=\left(F_{A} \tilde{\wedge} G_{B}\right)\left(x_{1}, y_{1}\right) \cap\left(F_{A} \tilde{\wedge} G_{B}\right)\left(x_{2}, y_{2}\right) .
\end{aligned}
$$

Hence, $F_{A} \tilde{\wedge} G_{B}$ is a soft intersection $K$-subalgebra over $U$.

Theorem 45. Let $\left\{\left(F_{i}\right)_{A_{i}} \mid i \in \Lambda\right\}$ be a family of soft intersection $K$-subalgebras (resp., soft intersection ideals) over $U$. Then, $\widetilde{\bigwedge}_{i \in \Lambda}\left(F_{i}\right)_{A_{i}}$ is a soft intersection $K$-subalgebra over $U$.

Proposition 46. Let $\mathscr{K}$ be a K-algebra and let $A$ be a $K$-subalgebra of $\mathscr{K}$. If $F_{A}$ and $G_{A}$ are soft intersection $K$ subalgebras over $U$. Then, $F_{A} \widetilde{\cap} G_{A}$ is a soft intersection $K$ algebra over $U$, where $F_{A} \widetilde{\cap} G_{A}$ is defined by $F_{A} \widetilde{\cap} G_{A}(x)=$ $F(x) \cap G(x)$ for all $x \in A$.

Proof. Let $x, y \in A$. Then,

$$
\begin{aligned}
\left(F_{A}\right. & \left.\tilde{\cap} G_{A}\right)(x \odot y) \\
& =F(x \odot y) \cap G(x \odot y) \\
& \supseteq(F(x) \cap F(y)) \cap(G(x) \cap G(y)) \\
& =(F(x) \cap G(x)) \cap(F(y) \cap G(y)) \\
& =\left(F_{A} \tilde{\cap} G_{A}\right)(x) \cap\left(F_{A} \tilde{\cap} G_{A}\right)(y) .
\end{aligned}
$$

Hence, $F_{A} \widetilde{\cap} G_{A}$ is a soft intersection $K$-subalgebras over $U$.

Theorem 47. Let $\left\{\left(F_{i}\right)_{A_{i}} \mid i \in \Lambda\right\}$ be a family of soft intersection $K$-subalgebras over $U$. Then, $\widetilde{\bigcap}_{i \in \Lambda}\left(F_{i}\right)_{A_{i}}$ is a soft intersection $K$-subalgebra over $U$.

Proposition 48. Let $\mathscr{K}$ be a $K$-algebra and let $A$ and $B$, be $K$-subalgebras of $\mathscr{K}$. If $F_{A}$ and $G_{B}$ are soft intersection $K$ subalgebras over $U$. Then $F_{A} \tilde{\times} G_{B}$, is a soft intersection $K$ algebra over $U$, where $F_{A} \tilde{\times} G_{B}$ is defined by $F_{A} \widetilde{\times} G_{B}(x, y)=$ $F(x) \times G(y)$ for all $(x, y) \in A \times B$.

Proof. Let $\left(x_{1}, y_{1}\right),\left(x_{2}, y_{2}\right) \in A \times B$. Then

$$
\begin{aligned}
& \left(F_{A} \tilde{\times} G_{B}\right)\left(\left(x_{1}, y_{1}\right) \odot\left(x_{2}, y_{2}\right)\right) \\
& \quad=\left(F_{A} \tilde{\times} G_{B}\right)\left(\left(x_{1} \odot x_{2}, y_{1} \odot y_{2}\right)\right)
\end{aligned}
$$

$$
\begin{aligned}
& =F\left(x_{1} \odot x_{2}\right) \times G\left(y_{1} \odot y_{2}\right) \\
& \supseteq\left(F\left(x_{1}\right) \cap F\left(x_{2}\right)\right) \times\left(G\left(y_{1}\right) \cap G\left(y_{2}\right)\right) \\
& =\left(F\left(x_{1}\right) \times G\left(y_{1}\right)\right) \cap\left(F\left(x_{2}\right) \times G\left(y_{2}\right)\right) \\
& =\left(F_{A} \tilde{\times} G_{B}\right)\left(x_{1}, y_{1}\right) \cap\left(F_{A} \tilde{\times} G_{B}\right)\left(x_{2}, y_{2}\right) .
\end{aligned}
$$
$U$.

Hence, $F_{A} \tilde{\times} G_{B}$ is a soft intersection $K$-subalgebras over

Theorem 49. Let $\left\{\left(F_{i}\right)_{A_{i}} \mid i \in \Lambda\right\}$ be a family of soft intersection $K$-subalgebras over $U$. Then, $\widetilde{\prod}_{i \in \Lambda}\left(F_{i}\right)_{A_{i}}$ is a soft intersection $K$-subalgebra over $U$.

Proposition 50. Let $\mathscr{K}$ be a K-algebra and let $A, B$ and $C$ be $K$-subalgebras of $\mathscr{K}$. If $F_{A}, G_{B}$ and $F_{C}$ are soft intersection $K$-subalgebras over $U, F_{A} \widetilde{\leq} G_{B}$, and $F_{C} \widetilde{\leq} G_{B}$, then $F_{A} \widetilde{\cap} F_{C} \widetilde{\leq} G_{B}$ over $U$.

Proof. Straightforward.

Definition 51 (see [25]). Let $F_{A}$ and $G_{B}$ be two soft sets over the common on universe $U$ and let $\varphi$ be a function from $A$ to $B$. Then, soft image of $F_{A}$ under $\varphi$ denoted by $\varphi\left(F_{A}\right)$ is a soft set over $U$ by

$\varphi(F)(y)= \begin{cases}\bigcup\{F(x): x \in A, \varphi(x)=y\} & \text { if } \varphi^{-1}(y) \neq \emptyset \\ \emptyset & \text { otherwise }\end{cases}$

for all $y \in B$, and soft preimage (or soft inverse image) of $G_{B}$ under $\varphi$ denoted by $\varphi^{-1}\left(G_{B}\right)$ is a soft set over $U$ by $\varphi^{-1}(G)(x)=G(\varphi(x))$ for all $x \in A$.

Proposition 52. Let $\mathscr{K}$ be a $K$-algebra and let $A$ be ideal of $\mathscr{K}$. If $F_{A}$ is a soft intersection $K$-subalgebra over $U$, then $A_{F}=$ $\{x \in A: F(x)=F(e)\}$ is a soft intersection $K$-subalgebra over $U$.

Theorem 53. Let $\mathscr{K}$ be a K-algebra and $A$ and $B$ ideals of $\mathscr{K}$. Let $\varphi$ be a $\mathscr{K}$-homomorphism from $A$ to $B$. If $G_{B}$ is soft intersection K-subalgebra over $U$, then $\varphi^{-1}\left(G_{B}\right)$ is a soft intersection $K$-subalgebra over $U$.

Proof. Let $x, y, z \in A$ and $m \in K$. Then,

$$
\begin{aligned}
\varphi^{-1} & \left(G_{B}\right)(x \odot y) \\
& =G_{B}(\varphi(x \odot y)) \\
& =G_{B}(\varphi(x) \odot \varphi(y)) \\
& \supseteq G(\varphi(x)) \cap G(\varphi(y)) \\
& =\varphi^{-1}(G)(x) \cap \varphi^{-1}(G)(y) .
\end{aligned}
$$

Hence, $\varphi^{-1}\left(G_{B}\right)$ is a soft intersection $K$-subalgebra over $U$. 
Theorem 54. Let $\mathscr{K}$ be a K-algebra and $A$ and $B$ ideals of $\mathscr{K}$, and let $\varphi$ be a $\mathscr{K}$-isomorphism from $A$ to $B$. If $F_{A}$ is a soft intersection $K$-subalgebra over $U$, then $\varphi\left(F_{A}\right)$ is a soft intersection $K$-subalgebra over $U$.

Proof. Since $\varphi$ is surjective, there exist $x, y \in A$ such that $a=\varphi(x)$ and $b=\varphi(y)$ for all $a, b \in B$. Then,

$$
\begin{aligned}
\varphi\left(F_{A}\right)(x \odot y) \\
=\cup\{F(z): z \in A, \varphi(z)=a \odot b\} \\
=\cup\{F(x \odot y): x, y \in A, \\
\varphi(x)=a, \varphi(y)=b\} \\
\supseteq \cup\{F(x) \cap F(y): x, y \in A, \\
\varphi(x)=a, \varphi(y)=b\} \\
\cap(\cup\{F(x): x \in A, \varphi(x)=a\}) \\
\cap(\cup\{F(y): y \in A, \varphi(y)=b\}) \\
=\varphi\left(F_{A}\right)(a) \cap \varphi\left(F_{A}\right)(b) .
\end{aligned}
$$

Hence, $\varphi\left(F_{A}\right)$ is a soft intersection $K$-subalgebra over $U$.

\section{Conclusions}

Presently, science and technology are featured with complex processes and phenomena for which complete information is not always available. For such cases, mathematical models are developed to handle various types of systems containing elements of uncertainty. A large number of these models are based on an extension of the ordinary set theory, namely, soft sets. In 1999, Molodtsov introduced the concept of soft set theory as a general mathematical tool for dealing with uncertainty and vagueness, and many researchers have created some models to solve problems in decision making and medical diagnosis. We have applied the concept of soft set theory to $K$-algebras and have investigated some of their properties. The natural extension of this research work is connected with the study of (i) fuzzy soft intersection $K$ algebras and (ii) roughness in $K$-algebras.

\section{Acknowledgment}

The authors are highly thankful to the referees for their valuable comments and suggestions.

\section{References}

[1] L. A. Zadeh, "Fuzzy sets," Information and Computation, vol. 8, pp. 338-353, 1965.
[2] L. A. Zadeh, "The concept of a linguistic variable and its application to approximate reasoning. I," vol. 8, pp. 199-249, 1975.

[3] D. Molodtsov, "Soft set theory first results," Computers \& Mathematics with Applications, vol. 37, no. 4-5, pp. 19-31, 1999.

[4] M. I. Ali, F. Feng, X. Y. Liu, W. K. Min, and M. Shabir, "On some new operations in soft set theory," Computers \& Mathematics with Applications, vol. 57, no. 9, pp. 1547-1553, 2009.

[5] H. Aktaş and N. Çağman, "Soft sets and soft groups," Information Sciences, vol. 177, no. 13, pp. 2726-2735, 2007.

[6] D. Chen, E. C. C. Tsang, D. S. Yeung, and X. Wang, "The parameterization reduction of soft sets and its applications," Computers \& Mathematics with Applications, vol. 49, no. 5-6, pp. 757-763, 2005.

[7] P. K. Maji, R. Biswas, and A. R. Roy, "Soft set theory," Computers \& Mathematics with Applications, vol. 45, no. 4-5, pp. 555-562, 2003.

[8] F. Feng, Y. B. Jun, X. Y. Liu, and L. Li, "An adjustable approach to fuzzy soft set based decision making," Journal of Computational and Applied Mathematics, vol. 234, no. 1, pp. 10-20, 2010.

[9] F. Feng, C. X. Li, B. Davvaz, and M. Irfan Ali, "Soft sets combined with fuzzy sets and rough sets: a tentative approach," Soft Computing, vol. 14, pp. 899-911, 2010.

[10] F. Feng, X. Y. Liu, V. Leoreanu-Fotea, and Y. B. Jun, "Soft sets and soft rough sets," Information Sciences, vol. 181, no. 6, pp. 11251137, 2011

[11] F. Feng, Y. B. Jun, and X. Zhao, "Soft semirings," Computers \& Mathematics with Applications, vol. 56, no. 10, pp. 2621-2628, 2008.

[12] U. Acar, F. Koyuncu, and B. Tanay, "Soft sets and soft rings," Computers \& Mathematics with Applications, vol. 59, no. 11, pp. 3458-3463, 2010.

[13] Y. B. Jun, "Soft BCK/BCI-algebras," Computers \& Mathematics with Applications, vol. 56, no. 5, pp. 1408-1413, 2008.

[14] O. Kazancı, S. Y1lmaz, and S. Yamak, "Soft sets and soft BCHalgebras," Hacettepe Journal of Mathematics and Statistics, vol. 39, no. 2, pp. 205-217, 2010.

[15] K. H. Dar and M. Akram, "On a $K$-algebra built on a group," Southeast Asian Bulletin of Mathematics, vol. 29, no. 1, pp. 4149, 2005.

[16] K. H. Dar and M. Akram, "On K-homomorphisms of $K$ algebras," International Mathematical Forum, vol. 2, no. 45-48, pp. 2283-2293, 2007.

[17] M. Akram and K. H. Dar, Generalized Fuzzy K-Algebras, VDM, 2010.

[18] M. Akram, K. H. Dar, Y. B. Jun, and E. H. Roh, "Fuzzy structures on $K(G)$-algebras," Southeast Asian Bulletin of Mathematics, vol. 31, no. 4, pp. 625-637, 2007.

[19] A. H. Handam, "Soft $K(G)$-algebras," Tamkang Journal of Mathematics, vol. 43, no. 2, pp. 203-213, 2012.

[20] Y. B. Jun and C. H. Park, "Fuzzy ideals of $K(G)$-algebras," Honam Mathematical Journal, vol. 28, no. 4, pp. 485-497, 2006.

[21] P. K. Maji, R. Biswas, and A. R. Roy, "Fuzzy soft sets," Journal of Fuzzy Mathematics, vol. 9, no. 3, pp. 589-602, 2001.

[22] M. Akram and F. Feng, "Soft intersection Lie algebras," Quasigroups and Related Systems, vol. 21, pp. 1-10, 2013.

[23] Y. B. Jun, K. J. Lee, and E. H. Roh, "Intersectional soft BCK/BCIideals," Annals of Fuzzy Mathematics and Informatics, vol. 4, no. 1, pp. 1-7, 2012. 
[24] J. Zhan and Y. Xu, "Soft lattice implication algebras based on fuzzy sets," Hacettepe Journal of Mathematics and Statistics, vol. 40, no. 4, pp. 483-492, 2011.

[25] N. Cagman, F. Citak, and K. Aktas, "Soft int-groups and its applications to group theory," Neural Computing and Applications, vol. 21, no. 1, supplement, pp. 151-158, 2012. 

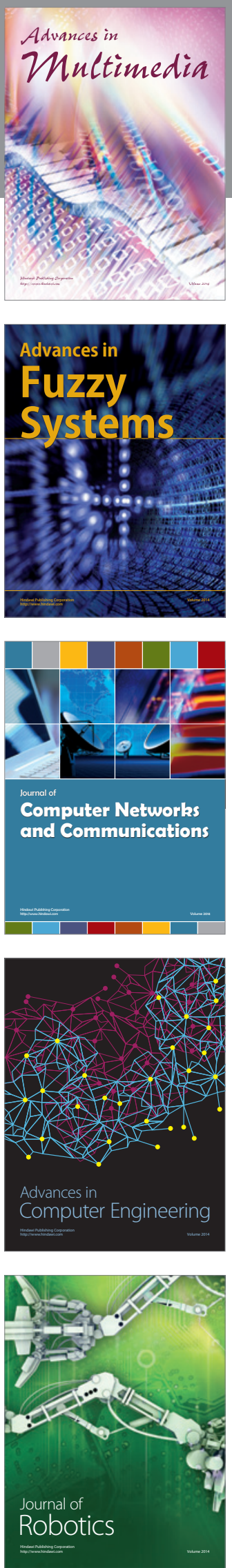

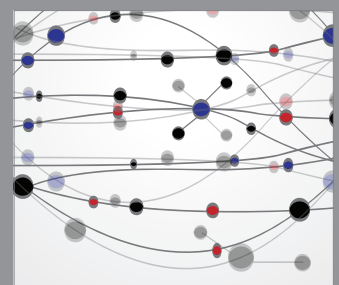

The Scientific World Journal
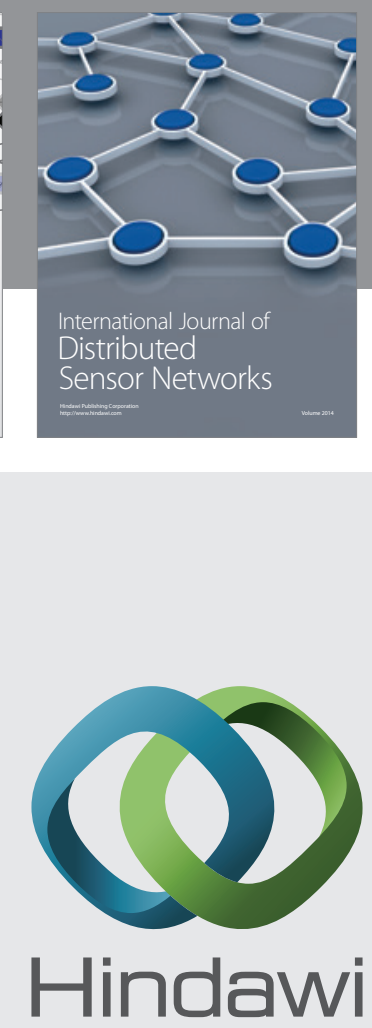

Submit your manuscripts at

http://www.hindawi.com
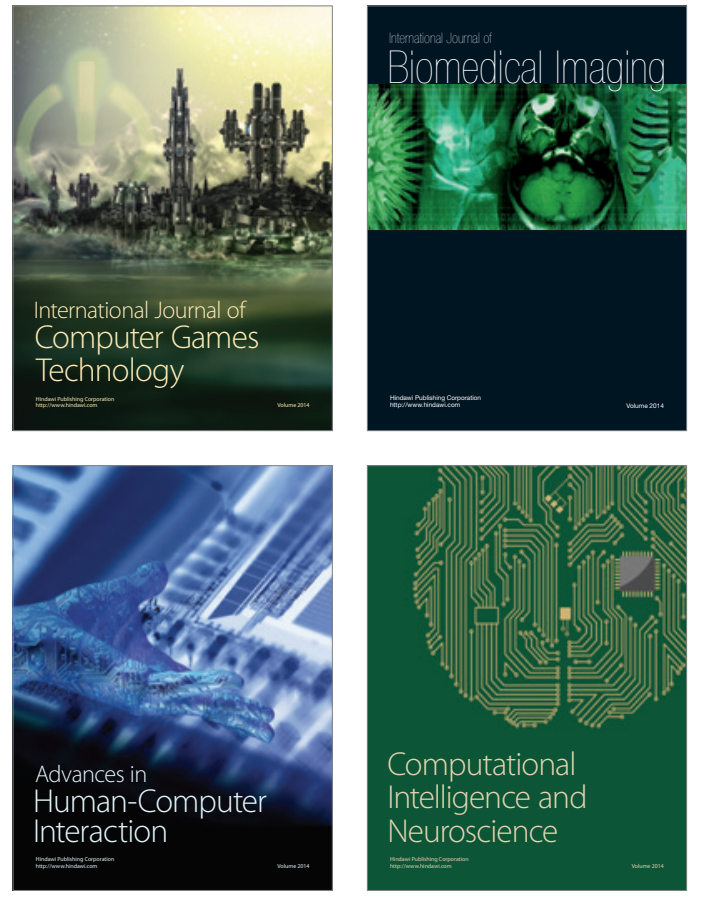
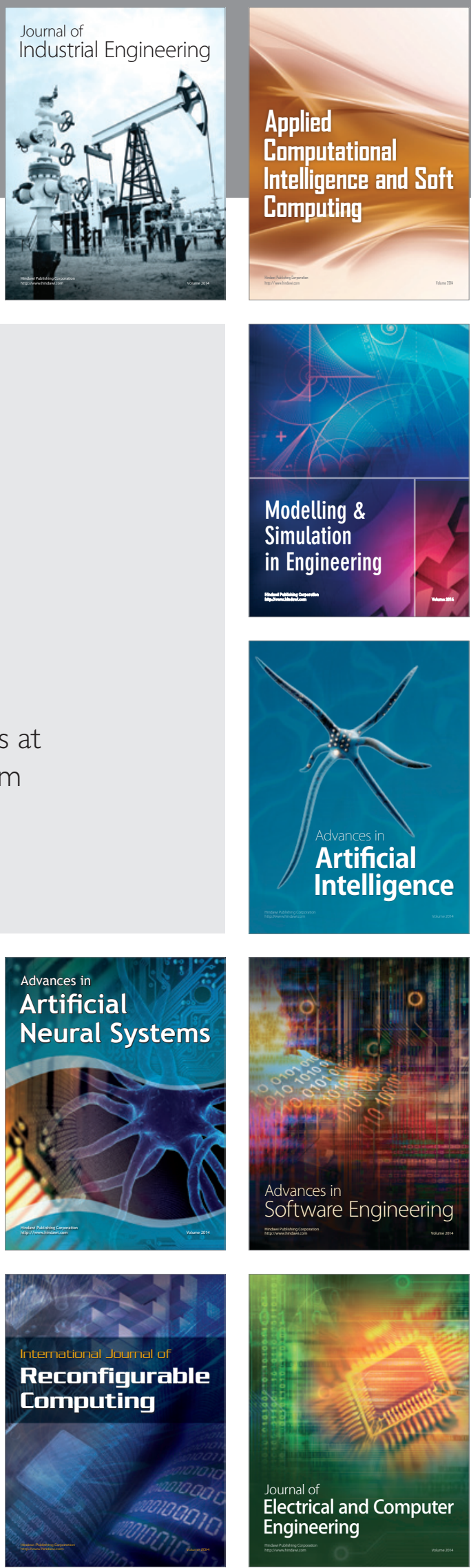